\title{
NORMAS DE VANCOUVER
}

\section{Resumen de los "Requisitos de Uniformidad del Comité Internacional de Directores de Revistas Médicas" (http://www.icmje.org/urm_main.html)}

\section{CUESTIONES PREVIAS ANTES DEL ENVÍO DE UN ORIGINAL}

Publicación redundante o duplicada

La publicación redundante o duplicada consiste en la publicación de un artículo que coincide sustancialmente con otro ya publicado o aceptado para publicar en otra revista. Los lectores de las revistas biomédicas deben tener la garantía de que aquello que están leyendo es original, a menos que se informe inequívocamente de que el artículo es una reedición, decidida por el autor o director de la revista.

Esta política no impide que una revista acepte un original rechazado por otras, o un trabajo completo con posterioridad a la publicación de un estudio preliminar en forma de resumen o póster presentado a un congreso científico, siempre que no haya sido publicado en su totalidad, o que se esté en ese momento considerando su publicación en las actas $u$ otro formato similar. El autor deberá informar al director de la revista acerca de cualquier presentación del documento a otras revistas, o cualquier trabajo anterior que pudiera considerarse publicación previa o duplicada de un trabajo idéntico o muy similar.

La divulgación preliminar, generalmente a través de los medios de comunicación, agencias gubernamentales, o fabricantes, de la información científica contenida en un artículo aceptado, pero aún sin publicar, representa una infracción de la política editorial que siguen muchas revistas. Esta divulgación puede defenderse cuando el artículo describa avances terapéuticos importantes o riesgos para la salud pública tales como efectos adversos de fármacos, vacunas, otros productos biológicos, o instrumentos médicos, o enfermedades de declaración obligatoria.

\section{Publicación secundaria aceptable}

La publicación secundaria en el mismo u otro idioma, especialmente en otros países, se justifica y puede ser beneficiosa si se dan las siguientes condiciones:

1. Que se disponga de la autorización de los directores de ambas revistas.

2. Se respetará la prioridad de la publicación original dejando transcurrir un intervalo de al menos una semana antes de la publicación de la segunda versión (salvo que ambos directores decidan otra cosa).

3. Que el artículo de la publicación secundaria se dirija a un grupo diferente de lectores, una versión resumida suele ser suficiente.

4. La versión secundaria debe reflejar fielmente los datos e interpretaciones de la original.
5. En una nota al pie de la primera página de la versión secundaria se debe informar de que el artículo ya ha sido publicado en todo o en parte y se debe hacer constar la referencia original. Un texto apropiado para dicha nota puede ser el que sigue: "El presente artículo se basa en un estudio publicado originalmente en (título de la revista y referencia completa)".

6. El permiso o autorización para la publicación secundaria deber gratuito.

Protección del derecho a la intimidad de los pacientes No debe infringirse el derecho a la intimidad de los pacientes sin su consentimiento informado. Por ello, no se publicará información de carácter identificativa en textos, fotografias e historiales clínicos, a menos que dicha información sea esencial desde el punto de vista científico y el paciente (familiares o tutor) haya dado su consentimiento por escrito para su publicación. El consentimiento al que nos referimos requiere que el paciente tenga acceso al documento original que se pretende publicar.

Guías de Pautas para el diseño específico de estudios Con frecuencia los investigadores en sus publicaciones omiten información importante. Se anima a los autores a que consulten las guías de pautas relativas al tipo de diseño concreto de su investigación. En los ensayos clínicos aleatorios los autores deben hacer referencia al cuestionario CONSORT. Esta guía proporciona un conjunto de recomendaciones mediante una lista de ítems a recoger y un diagrama de flujo del paciente.

\section{REQUISITOS PARA EL ENVÍO DE MANUSCRITOS VER INSTRUCCIONES PARA AUTORES \\ Carta de presentación}

1. Título del artículo. Debe ser conciso, pero informativo. En español y en inglés.

2. Autorías: los buscadores internacionales utilizan por defecto el último apellido. Se aconseja indicar un solo apellido o la unión de los apellidos con un guion.

3. Filiaciones: debe ser las que reconoce oficialmente cada institución.

4. Incluir identificador personal de investigación Publons (ResearcherlD) y/u Orcid.

5. Financiación. Origen del apoyo recibido ya sea en forma de subvenciones o de material para el trabajo.

6. Agradecimientos. (Ver más abajo).

7. Información adicional que se considere relevante.

8. Comentarios dirigidos al Director. Breve explicación del trabajo y de la relevancia del mismo. 


\section{Autoría}

Todas las personas que figuren como autores habrán de cumplir con ciertos requisitos para recibir tal denominación. Cada autor deberá haber participado en grado suficiente para asumir la responsabilidad pública del contenido del trabajo, exclusivamente en lo que se refiere a:

1. La concepción y el diseño del estudio, o recogida de los datos, o su análisis e interpretación.

2. La redacción del artículo o la revisión crítica de una parte sustancial de su contenido intelectual.

3. La aprobación final de la versión que será publicada.

Los requisitos 1, 2 y 3 tendrán que cumplirse simultáneamente.

El resto de personas que contribuyan al trabajo y que no sean los autores deben citarse en la sección de agradecimientos.

El orden de los autores dependerá de la decisión que de forma conjunta adopten los coautores. En todo caso, los autores deben ser capaces de explicar el mismo.

\section{Resumen y Palabras clave}

La segunda página incluirá un resumen. En él se indicarán los objetivos del estudio, los procedimientos básicos (la selección de los sujetos del estudio o de los animales de laboratorio, los métodos de observación y analíticos), los resultados más destacados (mediante la presentación de datos concretos y, a ser posible, de su significación estadística), y las principales conclusiones. Se hará hincapié en aquellos aspectos del estudio o de las observaciones que resulten más novedosos o de mayor importancia.

Tras el resumen los autores deberán presentar e identificar como tales, de 3 a 10 palabras clave que faciliten a los documentalistas el análisis documental del artículo y que se incluirán junto con el resumen. Utilícense para este fin los términos del Medical Subject Headings (MeSH) del Index Medicus; en el caso de que se trate de términos de reciente aparición que aún no figuren en el MeSH pueden usarse los nuevos términos. Se puede consultar una edición en español del MeSH elaborado por BIREME: "Descriptores de Ciencias de la Salud". Tanto el resumen como las palabras clave debe ir en español y en inglés

\section{Introducción}

Se indicará el propósito del artículo y se realizará de forma resumida una justificación del estudio. En esta sección del artículo, únicamente, se incluirán las referencias bibliográficas estrictamente necesarias y no se incluirán datos o conclusiones del trabajo.

\section{Métodos}

Describa con claridad la forma como fueron seleccionados los sujetos sometidos a observación o participantes en los experimentos (pacientes o animales de laboratorio, también los controles). Indique la edad, sexo y otras características destacadas de los sujetos. En el apartado de métodos se ha de especificar cuidadosamente el significado de los términos utilizados y detallar de forma exacta cómo se recogieron los datos (por ejemplo, qué expresiones se incluyen en la encuesta, si se trata de un cuestionario autoadministrado o la recogida se realizó por otras personas etc.).

Describa los métodos, aparataje (facilite el nombre del fabricante y su dirección entre paréntesis) y procedimientos empleados con el suficiente grado de detalle para que otros investigadores puedan reproducir los resultados. Se ofrecerán referencias de los métodos acreditados entre ellos los estadísticos; se darán referencias y breves descripciones de los métodos que aunque se hallen publicados no sean ampliamente conocidos; se describirán los métodos nuevos o sometidos a modificaciones sustanciales, razonando su utilización y evaluando sus limitaciones. Identifique con precisión todos los fármacos y sustancias químicas utilizadas, incluya los nombres genéricos, dosis y vías de administración.

En los ensayos clínicos aleatorios se aportará información sobre los principales elementos del estudio, entre ellos el protocolo (población a estudio, intervenciones o exposiciones, resultados y razonamiento del análisis estadístico), la asignación de las intervenciones (métodos de distribución aleatoria, de ocultamiento en la asignación a los grupos de tratamiento), y el método de enmascaramiento.

Cuando se trate de artículos de revisión, se ha de incluir una sección en la que se describirán los métodos utilizados para localizar, seleccionar, recoger y sintetizar los datos. Estos métodos se describirán también en el resumen del artículo.

\section{Ética}

Cuando se trate de estudios experimentales en seres humanos, indique si se siguieron las normas éticas del comité (institucional o regional) encargado de supervisar los ensayos en humanos y la declaración de Helsinki de 1975 modificada en 1983. No emplee, sobre todo en las ilustraciones, el nombre, ni las iniciales ni el número de historia clínica de los pacientes.

\section{Estadística}

Describa los métodos estadísticos con el suficiente detalle para permitir que un lector versado en el tema con acceso a los datos originales pueda verificar los resultados publicados. En la medida de lo posible, cuantifique los hallazgos y presente los mismos con los indicadores apropiados de error o de incertidumbre de la medición (como los intervalos de confianza). Se evitará la dependencia exclusiva de las pruebas estadísticas de verificación de hipótesis, tal como el uso de los valores $p$, que no aportan ninguna información cuantitativa importante. Analice los criterios de inclusión de los sujetos experimentales. Proporcione detalles sobre el proceso que se ha seguido en la distribución aleatoria. Describa los métodos de enmascaramiento utilizados. Indique las pérdidas de sujetos de observación (como los abandonos en un ensayo clínico). 
Siempre que sea posible, las referencias sobre el diseño del estudio y métodos estadísticos serán de trabajos vigentes (indicando el número de las páginas) en lugar de los artículos originales donde se describieron por vez primera. Especifique cualquier programa de ordenador, de uso común, que se haya empleado.

En la sección de métodos incluya una descripción general de los métodos empleados. Cuando en la sección de resultados resuma los datos, especifique los métodos estadísticos que se emplearon para analizarlos. Se restringirá el número de tablas y figuras al mínimo necesario para explicar el tema objeto del trabajo y evaluar los datos en los que se apoya. Use gráficos como alternativa a las tablas extensas. Defina los términos, abreviaturas y la mayoria de los símbolos estadísticos.

\section{Resultados}

Presente los resultados en el texto, tablas y gráficos siguiendo una secuencia lógica. No repita en el texto los datos de las tablas o ilustraciones; destaque o resuma tan sólo las observaciones más importantes.

\section{Discusión}

Haga hincapié en aquellos aspectos nuevos e importantes del estudio y en las conclusiones que se deriven de ellos. No debe repetir, de forma detallada, los datos u otras informaciones ya incluidas en los apartados de introducción y resultados. Explique en el apartado de discusión el significado de los resultados, las limitaciones del estudio, así como, sus implicaciones en futuras investigaciones. Se compararán las observaciones realizadas con las de otros estudios pertinentes.

Relacione las conclusiones con los objetivos del estudio, evite afirmaciones poco fundamentadas y conclusiones insuficientemente avaladas por los datos. En particular, los autores deben abstenerse de realizar afirmaciones sobre costes o beneficios económicos, salvo que en su artículo se incluyan datos y análisis económicos. No se citarán trabajos que no estén terminados. Proponga nuevas hipótesis cuando esté justificado, pero identificándolas claramente como tales. Podrán incluirse recomendaciones cuando sea oportuno.

\section{Agradecimientos}

Incluya la relación de todas aquellas personas que han colaborado pero que no cumplan los criterios de autoría, tales como, ayuda técnica recibida, ayuda en la escritura del manuscrito o apoyo general prestado. También se incluirá en los agradecimientos el apoyo financiero y los medios materiales recibidos.

Dado que los lectores pueden deducir que las personas citadas en los agradecimientos de alguna manera avalan los datos y las conclusiones del estudio, se obtendrá la autorización por escrito de las personas citadas en dicha sección.

\section{Referencias bibliográficas}

Los requisitos y orientaciones relativas a las referencias bibliográficas se encuentran al final de este resumen.

Tablas y gráficas

Sitúelas en el texto en el lugar que les correspondan. No presente las tablas en forma de fotografías. Numere las tablas consecutivamente en el orden de su primera citación en el texto y asígneles un breve título a cada una de ellas. En cada columna figurará un breve encabezamiento. Las explicaciones precisas se podrán en notas a pie de página, no en la cabecera de la tabla. En estas notas se especificarán las abreviaturas no usuales empleadas en cada tabla. Como llamadas para las notas al pie, utilícense los símbolos siguientes en la secuencia que a continuación se indica: ${ }^{*},+, \neq, q_{1}^{* *},+十, \neq \neq$, etc.

Identifique las medidas estadísticas de variación, tales como la desviación estándar o el error estándar de la media.

Asegúrese de que cada tabla esté citada en el texto.

\section{Unidades de medida}

Las medidas de longitud, talla, peso y volumen se deben expresar en unidades métricas (metro, kilogramo, litro) o sus múltiplos decimales.

Las temperaturas se facilitarán en grados Celsius y las presiones arteriales en milímetros de mercurio.

Todos los valores de parámetros hematológicos y bioquímicos se presentarán en unidades del sistema métrico decimal, de acuerdo con el Sistema Internacional de Unidades (SI).

\section{Abreviaturas y símbolos}

Utilice únicamente abreviaturas normalizadas. Evite las abreviaturas en el título y en el resumen. Cuando en el texto se emplee por primera vez una abreviatura, ésta irá precedida del término completo, salvo si se trata de una unidad de medida común.

\section{ENVÍO DEL MANUSCRITO A LA REVISTA}

Los manuscritos se acompañarán de una carta de presentación firmada por todos los autores (ya indicada anteriormente). 


\section{DECLARACIONES ADICIONALES}

Definición de una revista con sistema de revisión por expertos (peer-review)

Una revista con revisión por expertos (peer-review) es aquella que somete la mayoría de los artículos que publica a la evaluación de revisores, expertos en la materia, y que no forman parte del consejo editorial de la revista. El número y tipo de manuscritos revisados, el número de revisores, los procedimientos de evaluación y el uso que se haga de las opiniones de los revisores pueden variar $y$, por tanto, cada revista debería divulgar públicamente, en las normas de publicación o instrucciones para los autores, su política en esta cuestión en beneficio de los potenciales lectores y autores.

\section{Conflicto de intereses}

Se produce un conflicto de intereses en un artículo determinado cuando alguno de los que participan en el proceso de publicación (sea el autor, revisor o director) desarrollan actividades que pudieran condicionar el enjuiciamiento, tanto se produzca como no. Habitualmente, los conflictos de intereses más importantes consisten en la existencia de relaciones económicas con industrias (como empleado, consultoria, propiedad, honorarios, pruebas periciales) ya sea directamente 0 a través de familiares directos. Sin embargo, los conflictos de intereses pueden deberse a otras razones, tales como relaciones personales, competitividad académica o fanatismo intelectual.

Cuando se remite un manuscrito para su publicación, sea artículo o carta al director, los autores tienen la responsabilidad de reconocer y declarar la existencia de conflicto de intereses de tipo económico o de otro tipo que pudiera suponer un sesgo del trabajo. En el manuscrito se debe especificar todas las ayudas económicas recibidas que han hecho posible la realización del trabajo, así como otras relaciones de tipo económico o personal relacionadas con la misma.

\section{Ayuda de la Industria a proyectos específicos de investigación}

Los científicos tienen la obligación ética de publicar los resultados que sean fruto de su investigación. No deben llegar a acuerdos o establecer negociaciones que interfieran con su propio control sobre la decisión de publicar los artículos que escriban.

Cuando envien un manuscrito para su publicación, sea artículo o carta, es responsabilidad de los autores el reconocer y declarar los conflictos de intereses de tipo económico o de otro tipo que pudieran haber condicionado su trabajo. En el manuscrito deben reconocer toda la ayuda económica recibida en la realización del trabajo, así como otras conexiones económicas o personales del trabajo.

\section{El papel de la sección de correspondencia}

Todas las revistas biomédicas deben incluir una sección que incluya comentarios, preguntas o críticas sobre los artículos publicados y donde los autores de los artículos puedan responder. Generalmente, aunque no necesariamente, esta sección adopta el formato de Cartas al director.

\section{EJEMPLOS DE LAS REFERENCIAS BIBLIOGRÁFICAS}

\section{Artículos de revistas}

\section{Artículo estándar}

Autor/es. Título del artículo. Abreviatura internacional de la revista. año; volumen (número): página inicial-final del artículo.

Medrano MJ, Cerrato E, Boix R, Delgado-Rodríguez M. Factores de riesgo cardiovascular en la población española: metaanálisis de estudios transversales. Med Clin (Barc). 2005; 124(16): 606-12.

Más de seis autores: Si los autores fueran más de seis, se mencionan los seis primeros seguidos de la abreviatura et al.

Sosa Henríquez M, Filgueira Rubio J, López-Harce Cid JA, Díaz Curiel M, Lozano Tonkin C, del Castillo Rueda A et al. ¿Qué opinan los internistas españoles de la osteoporosis? Rev Clin Esp. 2005; 205(8): 379-82.

\section{Organización o equipo como autor}

Grupo de Trabajo de la SEPAR. Normativa sobre el manejo de la hemoptisis amenazante. Arch Bronconeumol. 1997; 33: $31-40$.

\section{Suplemento de un volumen}

Plaza Moral V, Álvarez Gutiérrez FJ, Casan Clará P, Cobos Barroso N, López Viña A, Llauger Rosselló MA et al. Comité Ejecutivo de la GEMA. Guía Española para el Manejo del Asma (GEMA). Arch Bronconeumol. 2003; 39(Supl 5): 1-42.

\section{Indicación del tipo de artículo}

Rivas Otero B de, Solano Cebrián MC, López Cubero L. Fiebre de origen desconocido y disección aórtica [carta]. Rev Clin Esp. 2003; 203; 507-8.

Castillo Garzón MJ. Comunicación: medicina del pasado, del presente y del futuro [editorial]. Rev Clin Esp. 2004; 204(4): 181-4.

\section{Artículo publicado electrónicamente antes que en versión impresa}

Nota: Las citas Epub ahead of print son referencias enviadas a PubMed por los editores de revistas que se publican en primera instancia online, adelantándose a la edición en papel. Posteriormente, cuando se publica en formato impreso, la referencia se modifica apareciendo los datos de la edición impresa, seguida de la electrónica Epub.

Sait KH, Ashour A, Rajabi M. Pregnancy outcome in non-gynecologic cancer. Arch Gynecol Obstet. 2004 Jun 2 [Epub ahead of print].

Sait KH, Ashour A, Rajabi M. Pregnancy outcome in non-gynecologic cancer. Arch Gynecol Obstet. 2005 Apr; 271(4): 346-9. Epub 2004 Jun 2.

\section{Libros}

\section{Autores individuales}

Autor/es. Título del libro. Edición. Lugar de publicación: Editorial; año. 
Jiménez Murillo L, Montero Pérez FJ. Compendio de Medicina de Urgencias: guía terapéutica. $2^{\text {a }}$ ed. Madrid: Elsevier; 2005.

Nota: La primera edición no es necesario consignarla. Si la obra estuviera compuesta por más de un volumen, debemos citarlo a continuación del título del libro Vol. 3.

Director(es), compilador(es) como autor

Espinás Boquet J. coordinador. Guía de actuación en Atención Primaria. 2a ed. Barcelona: Sociedad Española de Medicina; 2002.

Teresa E de, editor. Cardiología en Atención Primaria. Madrid: Biblioteca Aula Médica; 2003.

Nota: En la edición original figura "Editor" término inglés que se refiere al Editor Literario. En español este término debe traducirse como Director (de una revista) o Director, Compilador o Coordinador (de un libro).

\section{Capítulo de libro}

Autor/es del capítulo. Título del capítulo. En: Director/Coordinador/Editor del libro. Título del libro. Edición. Lugar de publicación: Editorial; año. página inicial-final del capítulo.

Mehta SJ. Dolor abdominal. En: Friedman $\mathrm{HH}$, coordinador. Manual de Diagnóstico Médico. $5^{\mathrm{a}}$ ed. Barcelona: Masson; 2004. p.183-90.

\section{Actas de congresos}

Segundo Congreso Nacional de la Asociación Española de Vacunología. Las Palmas de Gran Canaria; $13-15$ de Noviembre 2003. Madrid: Asociación Española de Vacunología; 2003.

\section{Comunicación presentada a un congreso}

Autor/es de la Comunicación/Ponencia. Título de la Comunicación/Ponencia. En: Título oficial del Congreso. Lugar de Publicación: Editorial; año. página inicial-final de la comunicación/ponencia.

Castro Beiras A, Escudero Pereira J. El Área del Corazón del Complejo Hospitalario "Juan Canalejo". En: Libro de Ponencias: V Jornadas de Gestión y Evaluación de Costes Sanitarios. Bilbao; Ministerio de Sanidad y Consumo, Gobierno Vasco; 2000. p. 12-22.

\section{Tesis Doctoral}

Autor. Título de la tesis [tesis doctoral]*. Lugar de publicación: Editorial; año.

Muñiz Garcia J. Estudio transversal de los factores de riesgo cardiovascular en población infantil del medio rural gallego [tesis doctoral]. Santiago: Servicio de Publicacións e Intercambio Científico, Universidade de Santiago; 1996.

*: en inglés: dissertation.
Otros trabajos publicados

Artículo de periódico

Autor del artículo*. Título del artículo. Nombre del periódico**. Día mes año; Sección $^{* * *}$ : página (columna) ${ }^{* * * *}$.

${ }^{*}$ Autor del artículo (si figurase).

** Los nombres de periódicos no se facilitan abreviados.

*** Si existiera identificada como tal.

**** Si aparece identificada.

Carrasco D. Avalado el plazo de cinco años para destruir parte de la HC. Diario Médico. Viernes 23 de julio de 2004; Normativa: 8.

\section{Documentos legales}

Leyes/Decretos/Ordenes....

Título de la ley/decreto/orden... (Nombre del Boletín Oficial, número, fecha de publicación). Estatuto Marco del personal estatutario de los servicios de salud. Ley 55/2003 de 16 de diciembre. Boletín Oficial del Estado, n 301, (17-12-2003).

Orde do 7 de xullo de 2004 pola que se crea a Comisión de Coordinación de Calidade da Consellería de Sanidade e do Servizo Galego de Saúde. Diario Oficial de Galicia, ${ }^{\circ}$ 138, (19 de xullo de 2004).

\section{Material electrónico}

Artículo de revista en Internet

Autor/es del artículo. Título del artículo. Nombre de la revista [revista en Internet] ${ }^{*}$ año [fecha de consulta] ${ }^{* *}$; volumen (número): [Extensión/páginas ${ }^{* *}$ ]. Dirección electrónica.

Francés I, Barandiarán M, Marcellán T, Moreno L. Estimulación psicocognoscitiva en las demencias. An Sist Sanit Navar [revista en Internet] 2003 septiembre-diciembre. [acceso 19 de octubre de 2005]; 26(3). Disponible en: http://www.cfnavarra.es/salud/anales/textos/vol26/n3/ revis2a.html

* Puede sustituirse por: [Revista on-line], [Internet], [Revista en línea]

** [acceso....], [consultado...], [citado...]

*** Si constasen.

\section{Monografía en Internet}

Autor/es o Director/Coordinador/Editor. Título [Monografía en Internet]*. Edición. Lugar de publicación: Editor; año [fecha de consulta]. Dirección electrónica.

Moraga Llop FA. Protocolos diagnósticos y terapéuticos en Dermatología Pediátrica. [Monografía en Internet] *. Madrid: Asociación Española de Pediatría; 2003 [acceso 19 de diciembre de 2005]. Disponible en: http://www. aeped.es/protocolos/dermatologia/index.htm

Zaetta JM, Mohler ER, Baum R. Indications for percutaneous interventional procedures in the patient with claudication. [Monografía en Internet]. Walthman (MA): UpToDate; 2005 [acceso 30 de enero de 2006]. Disponible en: http://www.uptodate.com/ 
Puede sustituirse por: [Monografía en línea], [Internet], [Libro en Internet]. Ejemplo de citación recomendado por Uptodate:

Zaetta, JM, Mohler, ER, Baum, R. Indications for percutaneous interventional procedures in the patient with claudication. In: UpToDate, Rose, BD (Ed), UpToDate, Waltham, MA, 2005.

Parte de una página de un sitio o sede Web

Título de la página [sede Web]*. Lugar de publicación: Editor; Fecha de publicación [fecha de actualización/revisión; fecha de acceso]. Título de la sección [número de páginas o pantallas]. Dirección electrónica.

Medicina Interna de Galicia [sede Web]*. Lugo: Sociedad Gallega de Medicina Interna; 2005 [acceso 19 de diciembre de 2005]. De Pablo Casas M, Pena Río JL. Guía para la prevención de complicaciones infecciosas relacionadas con catéteres intravenosos. Disponible en: https://meiga.info/guias/cateteres.pdf

American Medical Association [sede Web]*. Chicago: The Association; c1995-2002 [actualizado 5 de diciembre de 2005; acceso 19 de diciembre de 2005]. AMA Office of Group Practice Liaison; [aproximadamente 2 pantaIlas]. Disponible en: http://www.ama-assn.org/ama/pub/ category/1736.html

Uptodate. Smith CCh. Idiopathic hyperhidrosis [sede Web]. May 17, 2005 May 17, 2005. Uptodate Waltham, Massachusetts. http://www.uptodate.com/

Puede sustituirse por: [Página principal en Internet], [Internet], [Página de inicio en Internet], [Homepage], [Sede Web].

\section{RECOMENDACIONES PARA ESCRIBIR REFERENCIAS BIBLIOGRÁFICAS}

Las referencias o citas bibliográficas constituyen una sección destacada en un trabajo científico. La selección cuidadosa de documentos relevantes es un elemento que da solidez a exposición teórica del texto, a la vez que constituye una importante fuente de información para el lector.

Facilitamos una serie de indicaciones para elaborar las referencias bibliográficas basadas en los Requisitos de Uniformidad (estilo Vancouver).

- Las referencias deben numerarse consecutivamente según el orden en que se mencionen por primera vez en el texto. FARMACÉUTICOS COMUNITARIOS recomienda que se utilicen números arábigos entre corchetes: [1], [4,12], [6-9].

- Cuando hay más de una cita, éstas deben separarse mediante comas, pero si fueran correlativas, se menciona la primera y la última separadas por un guion.

- Cuando en el texto se menciona un autor, el número de la referencia se pone tras el nombre del autor. Si se tratase de un trabajo realizado por más de dos autores, se cita el primero de ellos seguido de la abreviatura "et al" y su número de referencia.
- Se incluirán sólo aquellas referencias consultadas personalmente por los autores.

- Se recomienda no citar revistas traducidas al español. Es aconsejable recuperar la cita de la versión original, por resultar el documento original más fidedigno.

- Los documentos que se citen deben ser actuales. FARMACÉUTICOS COMUNITARIOS recomienda que no deben de tener más de cinco años y preferiblemente que sean de los dos últimos. Se recurriría a citar documentos que tengan más años, por motivos históricos o si no se encontrasen referencias actualizadas como alternativa.

- Las referencias que se realicen de originales aceptados pero aún no publicados se indicará con expresiones del tipo "en prensa" o "próxima publicación"; los autores deberán obtener autorización escrita y tener constancia que su publicación está aceptada.

- Debe evitarse citar resúmenes, excepto que sea un motivo muy justificado. Se consultarán los documentos completos. Tampoco cite una "comunicación personal", salvo cuando en la misma se facilite información esencial que no se halla disponible en fuentes públicamente accesibles, en estos casos se incluirán entre paréntesis en el texto, el nombre de la persona y la fecha de la comunicación. En los artículos científicos, los autores que citen una comunicación personal deberán obtener la autorización por escrito.

- Los títulos de las revistas deben abreviarse según el estilo que utiliza la National Library of Medicine (NLM). Puede consultarse el Journals Database de PubMed. Para comprobar las abreviaturas de revistas españolas, puede consultarse el catálogo C17 (Catálogo colectivo de publicaciones periódicas de las Bibliotecas de Ciencias de la Salud Españolas). En el supuesto de no localizar una abreviatura, puede consultarse la List of serial title Word abbreviations Internacional conforme a la norma ISO 4, o bien el "The List of Title Word Abbreviations" de la agencia ISSN.

- Una vez finalizada la bibliografía, tiene que asegurarse de la correspondencia de las citas en el texto y el número asignado en la bibliografía.

\section{BIBLIOGRAFÍA}

International Committee of Medical Journal Editors. Uniform Requirements for Manuscripts Submitted to Biomedical Journals: Writing and Editing for Biomedical Publication. Updated October 2005. [Internet]. CMJE; 2005 [acceso 17 de diciembre de 2005]. Disponible en: http://www.icmje.org/

National Library of Medicine Recommended Formats for Bibliographic Citation [Internet]. Bethesda: National Library of Medicine; Diciembre 2003, [acceso 17 de diciembre de 2005]. Disponible en: https://collections.nIm.nih.gov/bookviewer?PID=nlm:nlmuid-9107575-bk\#page/1/mode/2up 